MITSUBISHI ELECTRIC RESEARCH LABORATORIES

http://www.merl.com

\title{
Model Predictive Control for Simultaneous Station Keeping and Momentum Management of Low-Thrust Spacecraft
}

\author{
Weiss, A.; Kalabić, U.; Di Cairano, S. \\ TR2015-062 July 01, 2015
}

\begin{abstract}
We propose a Model Predictive Control (MPC) policy for simultaneous station keeping and momentum management of a low-thrust nadir-pointing satellite in geostationary orbit around the Earth. The satellite is equipped with six electrically powered thrusters and three axisymmetric reaction wheels, which must be coordinated to control the satellite's orbital position and, concurrently, unload the wheels' stored angular momentum. The MPC policy enforces constraints that maintain the satellite in a tight latitude and longitude window and in a tight nadir-pointing attitude configuration, while minimizing the delta-v provided by the thrusters. The MPC policy exploits a prediction model of the environmental disturbance forces in order to significantly reduce the delta-v required for station keeping, and enforces constraints determined by the thruster configuration to select control forces and torques that can be generated by the propulsion system. We present numerical simulations of the control policy in closed-loop with the satellite nonlinear dynamics that validate the performance of the proposed design in terms of thruster usage and constraint enforcement.
\end{abstract}

2015 American Control Conference (ACC)

\footnotetext{
This work may not be copied or reproduced in whole or in part for any commercial purpose. Permission to copy in whole or in part without payment of fee is granted for nonprofit educational and research purposes provided that all such whole or partial copies include the following: a notice that such copying is by permission of Mitsubishi Electric Research Laboratories, Inc.; an acknowledgment of the authors and individual contributions to the work; and all applicable portions of the copyright notice. Copying, reproduction, or republishing for any other purpose shall require a license with payment of fee to Mitsubishi Electric Research Laboratories, Inc. All rights reserved.
} 



\title{
Model Predictive Control for Simultaneous Station Keeping and Momentum Management of Low-Thrust Satellites
}

\author{
Avishai Weiss $^{1}$, Uroš Kalabić ${ }^{2}$ and Stefano Di Cairano ${ }^{3}$
}

\begin{abstract}
We propose a Model Predictive Control (MPC) policy for simultaneous station keeping and momentum management of a low-thrust nadir-pointing satellite in geostationary orbit around the Earth. The satellite is equipped with six electrically powered thrusters and three axisymmetric reaction wheels, which must be coordinated to control the satellite's orbital position and, concurrently, unload the wheels' stored angular momentum. The MPC policy enforces constraints that maintain the satellite in a tight latitude and longitude window and in a tight nadir-pointing attitude configuration, while minimizing the delta-v provided by the thrusters. The MPC policy exploits a prediction model of the environmental disturbance forces in order to significantly reduce the delta-v required for station keeping, and enforces constraints determined by the thruster configuration to select control forces and torques that can be generated by the propulsion system. We present numerical simulations of the control policy in closed-loop with the satellite nonlinear dynamics that validate the performance of the proposed design in terms of thruster usage and constraint enforcement.
\end{abstract}

\section{INTRODUCTION}

A satellite in geostationary Earth orbit (GEO) is subject to various non-Keplerian forces and disturbance torques that affect its ability to maintain station and nadir-pointing attitude [1], [2]. To counteract these perturbations, geostationary satellites are generally equipped with thrusters for station keeping (SK) maneuvers and reaction wheels for disturbance torque absorption.

For station keeping, satellites in GEO traditionally use chemical propulsion systems that are manually commanded from the ground to fire roughly once every 2 weeks in order to compensate for secular and periodic perturbations that force the satellite outside of its station keeping window (or deadband box), that is, a rectangular box of longitude and latitude above the Earth [3]-[5]. While the SK maneuvers that a GEO satellite performs are small, the total $\Delta v$ required over typical satellite lifetimes of 12-15 years is substantial. Thus, in recent years, GEO satellite designers have begun to utilize electric propulsion systems in order to reduce the mass fraction of the satellite. While electric propulsion for satellite station keeping is not conceptually new [6], onboard power requirements and resistance in adopting the technology has hindered their implementation hitherto [7].

Electric thrusters have significantly higher specific impulse $I_{\mathrm{sp}}$ than conventional chemical thrusters, i.e. they generate

A. Weiss and S. Di Cairano are with Mitsubishi Electric Research Laboratories, Cambridge, MA. U. Kalabic is with the Department of Aerospace Engineering, University of Michigan, Ann Arbor, MI He was an intern at Mitsubishi Electric Research Laboratories during this work. 1 weiss@merl.com, ${ }^{2}$ kalabic@umich.edu, 3 dicairanodieee.org force more efficiently with respect to propellant mass, enabling increased satellite longevity, larger payloads, and/or cheaper orbital insertion. Conversely, electric propulsion produces relatively low thrust (on the order of GEO perturbation forces), necessitating continuous thruster firing to impart an equivalent impulse to that of chemical thrusters. To date, much of the research on low-thrust SK has been on openloop optimal maneuver design, see, e.g. [8], [9]. However, given the desire to place geostationary satellites in tight longitudinal windows as required for satellite co-location, the corresponding mission autonomy requirements, and the overall density of the GEO belt, developing the capability for fine closed-loop control is attractive. For surveys on classical station keeping, see [10], [11] and references therein.

In addition to orbital perturbations, geostationary satellites are disturbed by environmental torques that must be absorbed by onboard momentum exchange devices. The satellite, however, cannot absorb an arbitrary amount of external torque, where, in the case of reaction wheels, this is due to the fact that the wheels cannot spin at arbitrarily high rates. To prevent saturation of the wheels and subsequent loss of the desired satellite attitude, the wheels' stored angular momentum must be unloaded via the onboard thrusters, which, classically, is a manually commanded process [12]. As mass is a driving consideration in satellite design, and in order to reduce complexity and cost, it is desirable to utilize the same set of thrusters for both station keeping and momentum unloading. While orbital and attitude control are generally treated as decoupled problems, using the same set of thrusters for both objectives couples the dynamics via constraints on the thrusters' ability to provide concurrent forces and torques.

In this work we address the problem of autonomous simultaneous station keeping and momentum management using low thrust propulsion via closed-loop control. The salient features of this problem, i.e. the need for continuous control techniques, the generation of fuel efficient maneuvers, the tight permissible station keeping window, the stringent constraints on available thrust, and the coordination required between orbital and attitude control, make it an ideal candidate for the application of model predictive control (MPC).

Given the limited onboard computational power on most satellites, we focus on the application of linear-quadratic MPC, whose quadratic programs (QPs) can be solved quickly and efficiently in resource constrained hardware, see, e.g., [13] and references therein. We build a prediction model for the MPC policy based on the linearization of the satellite 
dynamics around their nominal operating condition, using Euler angles to represent the attitude of the satellite relative to the nadir-pointing local vertical, local horizontal (LVLH) frame, and the Clohessy-Wiltshire Hill $(\mathrm{CWH})$ equations to control the satellite's position and velocity relative to a target location on an ideal geostationary orbit. We incorporate disturbance prediction using analytic expressions for the major perturbation forces, enabling the MPC policy to intelligently use the orbital plane coupling of the $\mathrm{CWH}$ equations to achieve fuel efficient maneuvers. Finally, we impose constraints on the size of the station keeping window, and constrain the simultaneous forces and torques available to the satellite based on thruster magnitude limits. For validation of this technique, in the simulations we consider the numerical integration of the full nonlinear governing equations and perturbation forces to propagate the satellite state forward in time. The results show that MPC is able to achieve fuel efficient SK maneuvers while satisfying the many objectives and constraints.

The paper is organized as follows. In Section II we present the kinematics and dynamics of a nadir-pointing satellite in GEO and review the main orbital perturbations it experiences. In Section III we present the formulation of the optimization problem constraints and develop the MPC policy. Section IV highlights the proposed strategy on numerical examples. Finally, we provide concluding remarks and thoughts on future research directions in Section V.

\section{PRELIMINARIES}

In this section we review the nonlinear and linearized orbital and attitude dynamics of geostationary satellites and present analytical expressions for the main perturbations that affect satellites in GEO.

\section{A. Notation}

The vector $\vec{r}_{\mathrm{q} / \mathrm{p}}$ denotes the position of point $\mathrm{q}$ relative to point $\mathrm{p}$, the vector $\vec{v}_{\mathrm{q} / \mathrm{p} / \mathrm{x}}$ denotes the velocity of point $\mathrm{q}$ relative to point $\mathrm{p}$ with respect to frame $\mathrm{F}_{\mathrm{X}}$, and the vector $\vec{\omega}_{\mathrm{Y} / \mathrm{X}}$ denotes the angular velocity of frame $\mathrm{F}_{\mathrm{Y}}$ relative to frame $\mathrm{F}_{\mathrm{X}}$. Note that $\overrightarrow{(\cdot)}$ denotes a coordinatefree (unresolved) vector. All frames are orthogonal and right handed.

\section{B. Satellite Orbital Dynamics}

The relative position vector of a satellite with respect to a target location on an orbit is expressed as

$$
\delta \vec{r}=\delta x \hat{i}+\delta y \hat{j}+\delta z \hat{k},
$$

where $\delta x, \delta y$ and $\delta z$ are the components of the position vector of the satellite relative to the target location and $\hat{\imath}$, $\hat{\jmath}, \hat{k}$ are the unit vectors of Hill's frame $\mathrm{F}_{\mathrm{H}}$. Hill's frame has its $x$-axis along the orbital radius, $y$-axis orthogonal to the $x$-axis and in the orbital plane, and $z$-axis orthogonal to orbital plane.

The position vector of the satellite with respect to the center of the Earth is given by $\vec{r}=\vec{r}_{0}+\delta \vec{r}$, where $\vec{r}_{0}$ is the nominal orbital position vector. The nonlinear equation of motion for the satellite relative to inertial frame $\mathrm{F}_{\mathrm{E}}$ is given by

$$
\ddot{\vec{r}}=-\mu \frac{\vec{r}}{r^{3}}+\frac{1}{m} \vec{F}+\overrightarrow{a_{\mathrm{p}}},
$$

where $\vec{F}$ is the vector of external forces applied to the satellite by the thrusters, $\overrightarrow{a_{\mathrm{p}}}$ is the vector of perturbation accelerations, $r=|\vec{r}|, m$ is the mass of the satellite, and $\mu$ is Earth's gravitational constant.

For small maneuvers around a nominal circular orbit, for which $\delta r<<r$, the linearized CWH equations [14], [15] approximate the relative motion of the satellite as

$$
\begin{aligned}
\delta \ddot{x}-3 n^{2} \delta x-2 n \dot{y} & =\frac{F_{x}}{m}+a_{\mathrm{p}, x}, \\
\delta \ddot{y}+2 n \delta \dot{x} & =\frac{F_{y}}{m}+a_{\mathrm{p}, y}, \\
\delta \ddot{z}+n^{2} \delta z & =\frac{F_{z}}{m}+a_{\mathrm{p}, z},
\end{aligned}
$$

where $F_{x}, F_{y}, F_{z}$ are components of the thrust force vector, $a_{\mathrm{p}, x}, a_{\mathrm{p}, y}, a_{\mathrm{p}, z}$ are components of the perturbation acceleration vector, and $n=\sqrt{\frac{\mu}{R_{0}^{3}}}$ denotes the mean motion of the nominal orbit.

\section{Satellite Attitude Kinematics}

The satellite's attitude is determined by Poisson's equation, which is given by

$$
\dot{R}(t)=R(t) \omega^{\times}(t),
$$

where $\omega(t) \in \mathbb{R}^{3}$ is the angular velocity of the satellite bus frame $F_{B}$ with respect to the inertial frame $F_{E}$ resolved in the satellite frame, $\omega^{\times}(t)$ is the cross-product matrix of $\omega(t)$, and $R(t)=\mathcal{O}_{\mathrm{E} / \mathrm{B}}(t) \in \mathbb{R}^{3 \times 3}$ is the rotation matrix that transforms the inertial frame into the satellite frame resolved in the satellite frame.

The attitude $R$ of a nadir-pointing satellite on a circular geostationary orbit must follow an attitude trajectory given by a time-varying continuously differentiable rotation matrix $R_{\mathrm{d}}(t)=\mathcal{O}_{\mathrm{E} / \mathrm{L}}(t)$ so that the bus-fixed frame $F_{\mathrm{B}}$ aligns with the LVLH frame $\mathrm{F}_{\mathrm{L}}$ and, for $t \geq 0, R_{\mathrm{d}}(t)$ is given by

$$
\begin{aligned}
& \dot{R}_{\mathrm{d}}(t)=R_{\mathrm{d}}(t) \omega_{\mathrm{d}}^{\times}(t), \\
& R_{\mathrm{d}}(0)=R_{\mathrm{d} 0},
\end{aligned}
$$

where $\omega_{\mathrm{d}}(t)$ is the desired angular velocity, and, for a nadirpointing satellite in GEO, is given by a constant spin about the axis perpendicular to the orbital plane with a period of one sidereal day.

The error between $R(t)$ and $R_{\mathrm{d}}(t)$ is given in terms of the attitude-error rotation matrix

$$
\tilde{R} \triangleq R^{\mathrm{T}} R_{\mathrm{d}}=\mathcal{O}_{\mathrm{E} / \mathrm{B}}^{\mathrm{T}} \mathcal{O}_{\mathrm{E} / \mathrm{L}}=\mathcal{O}_{\mathrm{B} / \mathrm{L}}
$$

We parameterize the attitude-error rotation matrix $\tilde{R}$ using the set of 3-2-1 Euler angles $(\psi, \theta, \phi)$ as $\tilde{R}=$ $C_{1}(\phi) C_{2}(\theta) C_{3}(\psi)$, where $C_{1}, C_{2}$, and $C_{3}$ are elementary rotations about the $x, y$, and $z$-axes by the angles $\psi, \theta$, and $\phi$, respectively. The linearized relationship between $(\psi, \theta, \phi)$ 
and $\omega$ about $\omega_{\mathrm{d}}=\left[\begin{array}{lll}0 & -\mathrm{n} & 0\end{array}\right]^{\mathrm{T}}$ is given by

$$
\begin{aligned}
\delta \dot{\phi} & =\delta \omega_{1}+n \delta \psi, \\
\delta \dot{\theta} & =\delta \omega_{2}, \\
\delta \dot{\psi} & =\delta \omega_{3}-n \delta \phi .
\end{aligned}
$$

\section{Attitude Dynamics for a Satellite with Reaction Wheels}

Consider a satellite actuated by three axisymmetric wheels $\mathrm{w}_{1}, \mathrm{w}_{2}, \mathrm{w}_{3}$, each with moment of inertia $J_{\mathrm{w}_{i}}=$ $\operatorname{diag}\left(\alpha_{i}, \beta_{i}, \beta_{i}\right)$, attached to a rigid bus $\mathrm{b}$ with moment of inertia $J_{\mathrm{b}}$ in an orthogonal configuration aligned with the principal axes of the bus. Each wheel is mounted so that it rotates about one of its own principal axes passing through its own center of mass with angular rate $\nu_{i}$. Then, the attitude dynamics of the satellite are given by [16]

$$
\begin{aligned}
J_{\mathrm{sc}} \dot{\omega} & =\left(J_{\mathrm{sc}} \omega+J_{\alpha} \nu\right) \times \omega-J_{\alpha} \eta+\tau, \\
\dot{\nu} & =\eta,
\end{aligned}
$$

where $\nu \triangleq\left[\begin{array}{lll}\nu_{1} & \nu_{2} & \nu_{3}\end{array}\right]^{\mathrm{T}}$, the vector $\tau$ represents the torque applied to the satellite from the thrusters, $J_{\alpha} \triangleq$ $\operatorname{diag}\left(\alpha_{1}, \alpha_{2}, \alpha_{3}\right)$ is the moment of inertia of the reaction wheel array, and $J_{\mathrm{sc}}=J_{\mathrm{b}}+J_{\alpha}$ is the moment of inertia of the satellite bus and reaction wheel array.

The linearization of (7) about an equilibrium $y$-axis (principal axis) spin $\omega_{\mathrm{d}}$ with an angular rate corresponding to the mean motion $n$ of the orbit yields

$$
\begin{aligned}
J_{\mathrm{sc}_{1}} \dot{\delta} \omega_{1}= & -\left(J_{\mathrm{sc}_{2}}-J_{\mathrm{sc}_{3}}+\alpha_{2}-\alpha_{3}\right) n \delta \omega_{3} \\
& +n \alpha_{3} \delta \nu_{3}-\alpha_{1} \eta_{1}+\tau_{1}, \\
J_{\mathrm{sc}_{2}} \dot{\delta} \omega_{2}= & -\alpha_{2} \eta_{2}+\tau_{2} \\
J_{\mathrm{sc}_{3}} \dot{\delta} \omega_{3}= & -\left(J_{\mathrm{sc}_{1}}-J_{\mathrm{sc}_{2}}+\alpha_{1}-\alpha_{2}\right) n \delta \omega_{1} \\
& -n \alpha_{1} \delta \nu_{1}-\alpha_{3} \eta_{3}+\tau_{3}, \\
\delta \dot{\nu}_{1}= & \eta_{1} \\
\delta \dot{\nu}_{2}= & \eta_{2}, \\
\delta \dot{\nu}_{3}= & \eta_{3} .
\end{aligned}
$$

\section{E. Perturbations}

Keplerian orbits do not exist in practice. Without orbital correction maneuvers, satellites drift from their assigned orbital positions due to various perturbational forces. For satellites in GEO, the main perturbations are solar and lunar gravitational attraction, solar radiation pressure, and the anisotropic geopotential, that is, Earth's non-spherical gravitational field. Analytic expressions for these perturbation forces per unit mass, i.e., the disturbance accelerations, are given, respectively, by

$$
\begin{gathered}
\vec{a}_{\text {sun }}=\mu_{\text {sun }}\left(\frac{\vec{r}_{\text {sun/sc }}}{r_{\text {sun } / \mathrm{sc}}^{3}}-\frac{\vec{r}_{\text {sun } / \text { earth }}}{r_{\text {sun/earth }}^{3}}\right), \\
\vec{a}_{\text {moon }}=\mu_{\text {moon }}\left(\frac{\vec{r}_{\text {moon/sc }}}{r_{\text {moon } / \mathrm{sc}}^{3}}-\frac{\vec{r}_{\text {moon/earth }}}{r_{\text {moon/earth }}^{3}}\right), \\
\vec{a}_{\text {srp }}=C_{\text {srp }} \frac{S\left(1+c_{\mathrm{refl}}\right)}{2 m} \frac{\vec{r}_{\mathrm{sc} / \mathrm{sun}}}{r_{\mathrm{sc} / \mathrm{sun}}}
\end{gathered}
$$

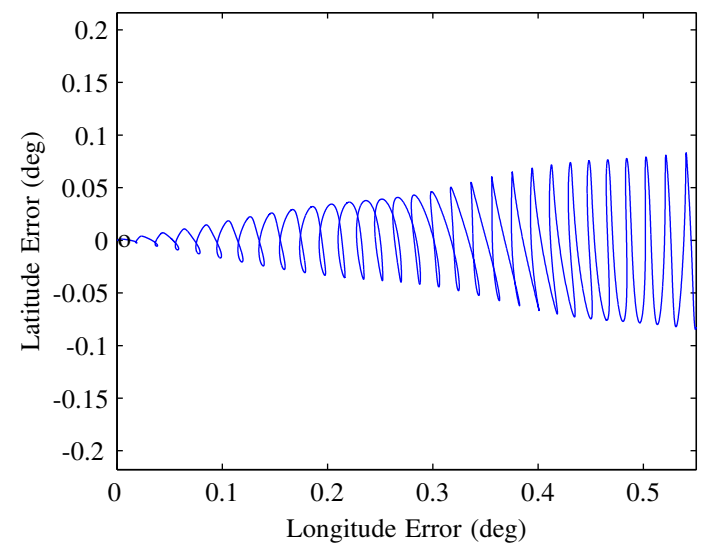

Fig. 1: Uncompensated motion for a $4000 \mathrm{~kg}$ satellite in geostationary orbit over one month.

$$
\vec{a}_{J_{2}}=\frac{3 \mu J_{2} \rho_{\mathrm{E}}^{2}}{2 r^{5}}\left(\left(5 \frac{\left(\vec{r} \cdot \hat{k}_{\mathrm{E}}\right)}{r^{2}}-1\right) \vec{r}-2\left(\vec{r} \cdot \hat{k}_{\mathrm{E}}\right) \hat{k}_{\mathrm{E}}\right),
$$

where $\mu_{\text {sun }}$ and $\mu_{\text {moon }}$ are the gravitational constants of the sun and moon, $C_{\text {srp }}$ is the solar radiation pressure constant, $S$ is the solar-facing surface area, $c_{\text {refl }}$ is the surface reflectance, $\rho_{\mathrm{E}}$ is Earth's equatorial radius, $\hat{k}_{\mathrm{E}}$ is the $z$-axis unit vector of the Earth-centered inertial frame $\mathrm{F}_{\mathrm{E}}$, and $J_{2}$ is the dominant coefficient in the considered geopotential perturbation model, where additional higher order terms are ignored.

The sum of the individual disturbance accelerations (9a)(9d)

$$
\vec{a}_{\mathrm{p}}=\vec{a}_{\text {sun }}+\vec{a}_{\text {moon }}+\vec{a}_{\text {srp }}+\vec{a}_{J_{2}}
$$

yields the total disturbance acceleration considered in (1). Figure 1 shows the uncompensated motion of the satellite subject to the aforementioned perturbations after one month.

Satellites are also subject to environmental torques such as those due to gravity gradients, solar radiation pressure, atmospheric drag, or the ambient magnetic field. In this work, we assume that these torques are absorbed by onboard reaction wheels via a nominal attitude control law and treat the resultant stored momentum as an initial condition.

\section{Controller Design}

The control requirements considered in this work for the simultaneous station keeping and momentum management of a geostationary satellite are:

1) Maintain the satellite in a tight station keeping window.

2) Maintain the satellite in a tight nadir-pointing configuration at all times, including during momentum unloading.

3) Unload the stored angular momentum from the reaction wheels, i.e., bring wheel speeds to 0 .

4) Limit the requested thruster magnitudes.

5) Minimize fuel consumption.

In order to handle the multitude of objectives, we design an MPC policy. MPC generates control actions by solving a receding-horizon finite-time optimal control problem based on a system model subject to pointwise-in-time state and control constraints and a user-defined cost function [17]. 
By using linearized equations of motion, linear equality and inequality constraints, and quadratic costs on the states and control actions, the MPC policy may be formulated as a $\mathrm{QP}$, which, given the limited computational resources onboard most satellites, can be solved quickly and efficiently [13]. In an MPC context, objectives 1, 2, and 4 naturally translate to constraints that can be incorporated in the MPC policy, whereas control objectives 3 and 5 are handled by appropriate selection of the MPC cost function.

\section{A. State Space Model}

We form a state-space model of the combined orbital dynamics (2), attitude kinematics (6), and attitude dynamics (8), to be used as a prediction model in the MPC policy. The model is given by

$$
\dot{x}(t)=A_{\mathrm{c}} x(t)+B_{\mathrm{c}} u(t),
$$

where

$$
\begin{aligned}
x= & {\left[\begin{array}{lllllllll}
\delta x & \delta y & \delta z & \delta \dot{x} & \delta \dot{y} & \delta \dot{z} & \delta \phi & \delta \theta & \delta \psi \\
\delta \omega_{1} & \delta \omega_{2} & \delta \omega_{3} & \delta \nu_{1} & \delta \nu_{2} & \delta \nu_{3}
\end{array}\right]^{\mathrm{T}}, } \\
u= & {\left[\begin{array}{lllllllll}
F_{x} & F_{y} & F_{z} & \eta_{1} & \eta_{2} & \eta_{3} & \tau_{1} & \tau_{2} & \tau_{3}
\end{array}\right]^{\mathrm{T}} . }
\end{aligned}
$$

Assuming a sampling period of $\Delta T$ sec, we discretize (11) which yields

$$
x_{k+1}=A x_{k}+B u_{k},
$$

where $x_{k}$ is the state at time step $k \in Z^{+}, u_{k}$ is the control vector at the time step $k \in Z^{+}$, and $A=\exp \left(A_{\mathrm{c}} \Delta T\right), B=$ $\int_{0}^{\Delta T} \exp \left(A_{c}(\Delta T-\tau)\right) d \tau B_{\mathrm{c}}$ are the discretized matrices obtained based on the continuous-time system realization $\left(A_{c}, B_{c}\right)$ in (11).

We augment (14) with a prediction model of the disturbance accelerations, obtaining

$$
x_{k+1}=A x_{k}+B u_{k}+\mathcal{O}_{\mathrm{H} / \mathrm{E}} a_{\mathrm{p}, k},
$$

where $a_{\mathrm{p}, k}$ is the total disturbance acceleration predicted at time step $k$ based on propagation of the desired nominal orbit, and $\mathcal{O}_{\mathrm{H} / \mathrm{E}}$ is the rotation matrix that transforms the components of $a_{\mathrm{p}, k}$ from the inertial frame into the components of the same acceleration in Hill's frame. The addition of the disturbance prediction in (15) enables the MPC policy to exploit natural relative motion dynamics, rather than propulsion, against the disturbance acceleration.

Remark 1. We use the nominal orbit for disturbanceacceleration prediction in (15) due to the nonlinearity of the analytical expressions in (9). Since the nominal orbit is known in advance, $a_{\mathrm{p}, k}$ can be predicted. As the spacecraft position is to be constrained in a tight station keeping window by objective 1 , the difference in the disturbance accelerations at the nominal orbital position and at the true satellite position is assumed to be negligible.

\section{B. Constraints}

Satellite position constraints may be imposed on $\delta y$ and $\delta z$, corresponding to a station keeping box using the relations

$$
|\delta y| \leq r_{0} \tan \left(\lambda_{1, \max }\right)
$$

$$
|\delta z| \leq r_{0} \tan \left(\lambda_{2, \max }\right),
$$

where $\lambda_{1, \max }$ is the maximum tolerable longitude error, and $\lambda_{2, \max }$ is the maximum tolerable latitude error.

We assume that the satellite is equipped with six dual-axis thrusters. Define $T \triangleq\left[\begin{array}{llllll}T_{1} & T_{2} & T_{3} & T_{4} & T_{5} & T_{6}\end{array}\right]$, where $T_{i}$ is the force exerted by each dual-axis thruster. We set constraints on the individual thruster magnitudes, i.e.,

$$
\|T\|_{\infty} \leq T_{\max },
$$

and relate them to constraints on the control input forces $F$ and torques $\tau$ via the force-torque map

$$
\left[\begin{array}{cc}
\mathcal{O}_{\mathrm{L} / \mathrm{H}} & 0 \\
0 & I
\end{array}\right]\left[\begin{array}{l}
F \\
\tau
\end{array}\right]=\left[\begin{array}{cc}
\Gamma & \Gamma \\
L & -L
\end{array}\right] T .
$$

Combining (17) and (18) yields

$$
\left\|\left[\begin{array}{cc}
\Gamma & \Gamma \\
L & -L
\end{array}\right]^{-1}\left[\begin{array}{cc}
\mathcal{O}_{\mathrm{L} / \mathrm{H}} & 0 \\
0 & I
\end{array}\right]\left[\begin{array}{l}
F \\
\tau
\end{array}\right]\right\|_{\infty} \leq T_{\max },
$$

which is enforced on the control input vector $u$ and accomplishes objective 4. Constraint (19) effectively couples (2) with (6),(8), i.e., the propulsion system has to generate both forces for orbital control and torques for attitude control.

In (18) we assume $\tilde{R}=\mathcal{O}_{\mathrm{B} / \mathrm{L}} \approx I$, i.e., that the attitude error is small and that the satellite is in the nominal nadirpointing configuration. This approximation is required to make the constraints linear. We further note that validity of this approximation is enforced by the attitude error constraint as follows.

We constrain the Euler angles $(\delta \phi, \delta \theta, \delta \psi)$ to be within a small tolerance as required by objective 2 ,

$$
|\delta \phi| \leq \delta \phi_{\max }, \quad|\delta \theta| \leq \delta \theta_{\max }, \quad|\delta \psi| \leq \delta \psi_{\max },
$$

in order to maintain a nadir-pointing configuration as usually required for operational purposes by geostationary satellites, and which also ensures that the linearization (6),(8) correctly approximates (3),(7).

\section{MPC Policy}

We consider the MPC policy that at any $t \in \mathbb{Z}_{0+}$ solves the finite-horizon optimal control problem

$$
\begin{array}{ll}
\min _{U} & x_{N}^{\mathrm{T}} P x_{N}+\sum_{k=1}^{N-1} x_{k}^{\mathrm{T}} Q x_{k}+u_{k}^{\mathrm{T}} R u_{k}, \\
\text { s.t. } & x_{k+1}=A x_{k}+B u_{k}+\mathcal{O}_{\mathrm{H} / \mathrm{E}} a_{\mathrm{p}, k}, \\
& x_{0}=x(t), \\
& T_{\min } \leq D u_{k} \leq T_{\max }, \\
& \delta y_{\min } \leq \delta y \leq \delta y_{\max }, \\
& \delta z_{\min } \leq \delta z \leq \delta z_{\max }, \\
& \delta \phi_{\min } \leq \delta \phi_{k} \leq \delta \phi_{\max }, \\
& \delta \theta_{\min } \leq \delta \theta_{k} \leq \delta \theta_{\max }, \\
& \delta \psi_{\min } \leq \delta \psi_{k} \leq \delta \psi_{\max },
\end{array}
$$

where $N$ is the control and prediction horizon, $U=$ $\left[u_{0}, \ldots, u_{N-1}\right], Q, R$ are specified state and control weight matrices, $D$ is the matrix that enforces the concurrently available forces and torques as in (19), and $P$ is the terminal 
state weighting matrix determined from the solution of the Discrete Algebraic Riccati Equation (DARE) for the unconstrained infinite horizon problem. The policy applies $u(t)=u_{0}^{*}$ to the satellite, where $\left[u_{0}^{*}, \ldots, u_{N-1}^{*}\right]$ is the optimal solution of (21), and repeats the procedure at time $t+1$, with $x(t+1)$ as a new initial condition.

The horizon $N$ is selected so that the MPC policy can predict and exploit the periodicity present in the dynamics and perturbations, and we verify that more than half an orbit is required. The state and control weight matrices $Q$ and $R$ are selected to aggressively minimize fuel consumption and unload the reaction wheels' stored angular momentum, which accomplishes objectives 3 and 5 .

Consistent with the standard MPC approach, we employ a terminal penalty matrix $P$ based on the solution to the DARE to guarantee local stability of the target equilibrium. Near the origin, where constraints are inactive, and in the absence of disturbance prediction, the solution of (21) is equivalent to that of an LQR controller. The maximum constraint admissible set $O^{\infty}$ of the LQR controller under constraints is a guaranteed domain of attraction for the MPC controller [17]. We note that recursive feasibility of (21) is guaranteed by relaxing state constraints into soft constraints by a slack variable. The finite-horizon optimal control problem (21) can be formulated as a quadratic program and solved by low complexity algorithms.

\section{Simulations}

We consider a $7.5 \times 5 \times 5 \mathrm{~m}$ satellite of mass $m=4000$ $\mathrm{kg}$ and inertia $J_{\mathrm{sc}}=\operatorname{diag}(1.7 e 4,2.7 e 4,2.7 e 4) \mathrm{kg} \cdot \mathrm{m}^{2}$ in geostationary orbit, i.e. $r_{0}=42164 \mathrm{~km}$, around the Earth. The satellite is constrained to a station keeping window of \pm 0.01 degrees longitude and latitude, while the maximum error allowed in the Euler angles is \pm 0.02 degrees. The satellite is propelled by six thrusters with $T_{\max }=0.1 \mathrm{~N}$. For this simulation,

$$
L=\left[\begin{array}{ccc}
0 & 2.5 & 0 \\
0 & 0 & 2.5 \\
3.75 & 0 & 0
\end{array}\right], \quad \Gamma=\left[\begin{array}{lll}
0 & 0 & 1 \\
1 & 0 & 0 \\
0 & 1 & 0
\end{array}\right]
$$

Each reaction wheel inertia about its rotation axis $\alpha_{i}$ is 0.8 $\mathrm{kg} \cdot \mathrm{m}^{2}$. Let $C_{\mathrm{srp}}=9.1 e-6 \mathrm{~N} / \mathrm{m}^{2}, S=200 \mathrm{~m}^{2}$, and $c_{\text {refl }}=$ 0.6 .

We consider two simulation scenarios: (i) annual station keeping simulation, to highlight the ability of the MPC policy to generate trajectories that are comparable with carefully designed (open-loop) station keeping techniques in terms of $\Delta v$ usage, and (ii) an aggressive station keeping and momentum unloading simulation, to highlight the MPC policy's ability to utilize the same set of thrusters, operating at their constraint limits, in order to satisfy both the force and torque requirements of station keeping and momentum unloading, respectively. We stress that, although the MPC policy uses a linearized dynamics model, all closed-loop simulations are fully nonlinear.

First we show an annual station keeping simulation, where we show that MPC is capable of producing fuel-efficient

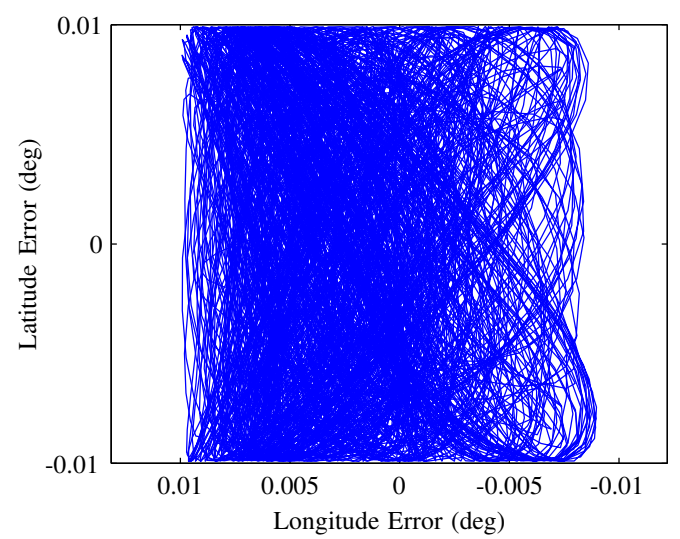

(a) Annual latitude and longitude
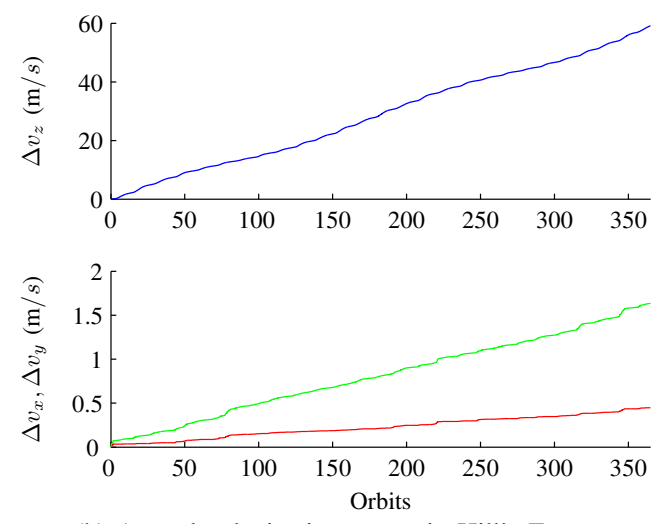

(b) Annual velocity increment in Hill's Frame.

Fig. 2: Annual station keeping simulation.

station keeping. In order to simulate an entire year, we discretize the dynamics with a sampling period $\Delta T=$ 3600 s and we set the MPC prediction horizon $N=15$. Figure $2 \mathrm{a}$ shows a simulation of the latitude and longitude error over one year. The satellite remains within the tight station keeping window of \pm 0.01 degrees. Figure $2 b$ shows the annual velocity increment $\Delta v$. In the out-of-plane $z$ direction, $\Delta v_{z}=59 \mathrm{~m} / \mathrm{s} /$ year. In the orbital plane, $\Delta v_{y}=$ $1.6 \mathrm{~m} / \mathrm{s} /$ year and $\Delta v_{x}=0.45 \mathrm{~m} / \mathrm{s} /$ year. We note that even though the station keeping window is about one order of magnitude smaller than traditional windows, the MPC policy is comparable in fuel usage to traditional SK techniques that employ larger windows. For instance in [7], it is claimed that for a positional accuracy of 0.05-0.1 degrees, 'NorthSouth' Station Keeping (NSSK), i.e. out-of-orbital-plane SK, requires between 41 and $51 \mathrm{~m} / \mathrm{s} /$ year, and 'East-West' Station Keeping (EWSK) requires $1.9 \mathrm{~m} / \mathrm{s} /$ year.

Next we show an aggressive station keeping and momentum unloading simulation, where we show that MPC is capable of using the same set of thrusters to generate both orbital forces and attitude torques. The satellite has an initial orbital displacement of $\delta x=\delta y=\delta x=1 \mathrm{~km}$, and an initial reaction wheel spin rate $\delta \nu_{i}=100 \mathrm{rad} / \mathrm{s}$. Since this maneuver occurs over a much shorter time period, we discretize the dynamics with a sampling period $\Delta T=600$ s. Figure $3 \mathrm{a}$ shows that the Euler angles remain within their 

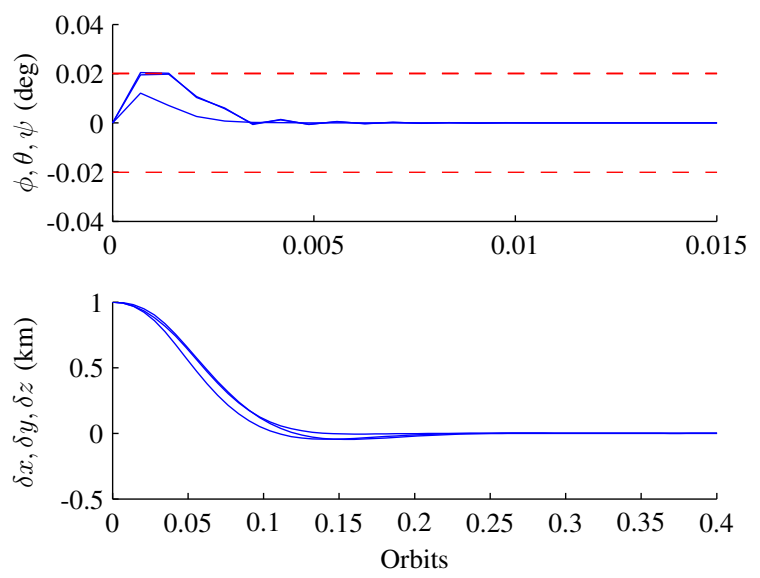

(a) Time histories of Euler angles and relative positions.
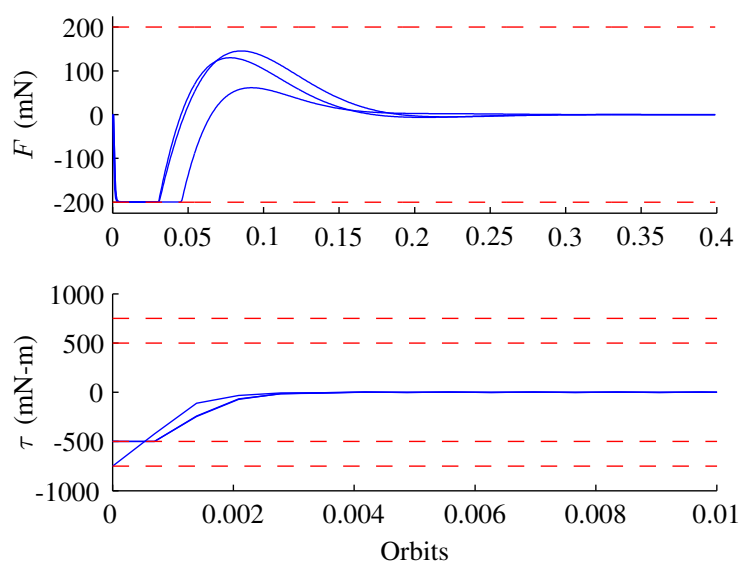

(b) Time histories of spacecraft forces and torques.
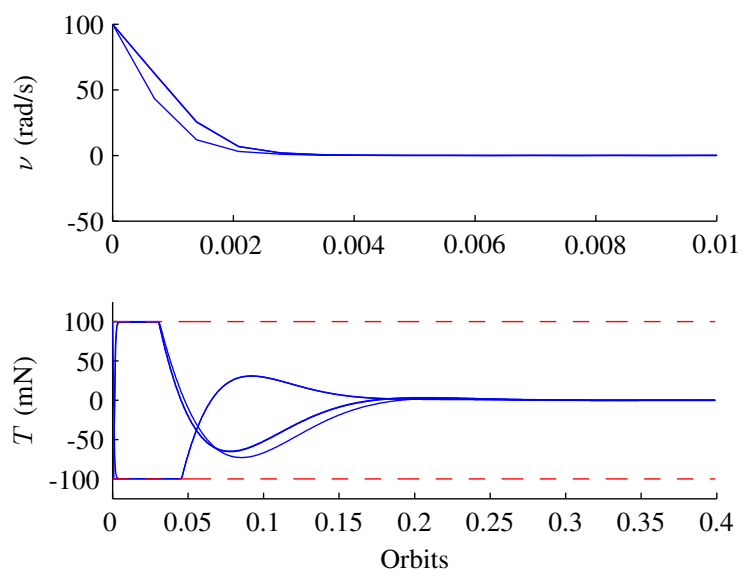

(c) Time histories of spacecraft reaction-wheel angular velocities and thruster magnitudes.

Fig. 3: Simulation of aggressive station keeping and momentum unloading maneuver.

limits during momentum unloading and that the satellite position is aggressively regulated. In Figure $3 \mathrm{~b}$, we see that forces and torques cannot concurrently be at their individual limits. When the thrusters generate maximum torque, there is no available thrust to force the satellite towards its nominal orbital position. Figure $3 \mathrm{c}$ shows that individual thrusters ride their magnitude constraints and transition from pure torque to pure force and that the reaction wheels' stored angular momentum is unloaded.

\section{CONCLUSIONS}

With future geostationary satellite missions requiring smaller station keeping windows, open-loop (manual) corrections may become unsustainable [18]. The introduction of autonomous closed-loop feedback control will increase robustness, safety and reliability of satellite station keeping, while reducing operational costs and risk of collisions. In this paper we have developed an MPC policy for autonomous closed-loop station keeping and momentum management of a geostationary satellite. We have shown that the MPC policy satisfies the specification constraints with a fuel consumption comparable to that of carefully designed open-loop maneuvers, which, however, have significantly larger station keeping windows. In addition, the proposed MPC policy is capable of concurrently performing station keeping and momentum unloading using the same set of thrusters.

\section{REFERENCES}

[1] R. R. Bate, D. D. Mueller, and J. E. White, Fundamentals of Astrodynamics. Dover publications, 1971.

[2] P. C. Hughes, Spacecraft Attitude Dynamics. John Wiley \& Sons Inc, 1986.

[3] E. M. Soop, Handbook of Geostationary Orbits. Springer, 1994.

[4] D. Losa, M. Lovera, R. Drai, T. Dargent, and J. Amalric, "Electric station keeping of geostationary satellites: a differential inclusion approach," in Proc. Conf. Dec. Contr. IEEE, 2005, pp. 7484-7489.

[5] D. Chu, D. Early, D. Freesland, et al., "GOES-R station-keeping and momentum management," in Proc. of the AAS Rocky Mountain Guidance and Contr. Conf., AAS 06-046, 2006.

[6] K. E. Clark, "Survey of electric propulsion capability," Journal of Spacecraft and Rockets, vol. 12, no. 11, pp. 641-654, 1975.

[7] M. Martinez-Sanchez and J. E. Pollard, "Spacecraft electric propulsion-an overview," Journal of Propulsion and Power, vol. 14 no. 5, pp. 688-699, 1998.

[8] T. N. Edelbaum, "Optimum low-thrust rendezvous and station keeping," AIAA Journal, vol. 2, no. 7, pp. 1196-1201, 1964.

[9] A. Sukhanov and A. Prado, "On one approach to the optimization of low-thrust station keeping manoeuvres," Advances in Space Research, vol. 50, no. 11, pp. 1478-1488, 2012.

[10] C. Chao and J. Baker, "On the propagation and control of geosynchronous orbits," Journal of the Astronautical Sciences, vol. 31, pp. 99-115, 1983.

[11] S. K. Shrivastava, "Orbital perturbations and stationkeeping of communication satellites," Journal of Spacecraft and Rockets, vol. 15, no. 2, pp. 67-78, 1978

[12] G. R. Cross, M. A. Potter, J. D. Whitehead, and J. T. Smith, "TDRSS momentum unload planning," Telematics and Informatics, vol. 8, no. 4, pp. 253-265, 1991.

[13] S. Di Cairano, M. Brand, and S. A. Bortoff, "Projection-free parallel quadratic programming for linear model predictive control," International Journal of Control, vol. 86, no. 8, pp. 1367-1385, 2013.

[14] W. Clohessy and R. Wiltshire, "Terminal guidance system for satellite rendezvous," J. Aerospace Sci, vol. 27, no. 9, pp. 653-658, 1960.

[15] B. Wie, Space Vehicle Dynamics and Control, 2nd ed. Reston, VA: AIAA, 2008.

[16] A. Weiss, I. Kolmanovsky, D. S. Bernstein, and A. Sanyal, "Inertiafree spacecraft attitude control using reaction wheels," Journal of Guidance, Control, and Dynamics, vol. 36, no. 5, pp. 1425-1439, 2013.

[17] S. Keerthi and E. G. Gilbert, "Optimal infinite-horizon feedback laws for a general class of constrained discrete-time systems: Stability and moving-horizon approximations," Journal of optimization theory and applications, vol. 57, no. 2, pp. 265-293, 1988.

[18] J. R. Wertz and W. J. Larson, Space mission analysis and design. Microcosm, 1999. 